\title{
Numerical Research on Convective Heat Transfer and Resistance Characteristics of Turbulent Duct Flow Containing Nanorod-Based Nanofluids
}

\author{
Fangyang Yuan $\mathbb{D}^{1,2}$ Jianzhong Lin $\mathbb{D}^{3},{ }^{3}$ and Jianfeng $Y u^{1}$ \\ ${ }^{1}$ School of Mechanical Engineering, Jiangnan University, Wuxi, China \\ ${ }^{2}$ Jiangsu Key Laboratory of Green Process Equipment, Changzhou University, Changzhou, China \\ ${ }^{3}$ School of Aeronautics and Astronautics, Zhejiang University, Hangzhou, China
}

Correspondence should be addressed to Jianzhong Lin; mecjzlin@public.zju.edu.cn

Received 25 October 2017; Accepted 21 January 2018; Published 6 March 2018

Academic Editor: Carlos R. Cabrera

Copyright ( 12018 Fangyang Yuan et al. This is an open access article distributed under the Creative Commons Attribution License, which permits unrestricted use, distribution, and reproduction in any medium, provided the original work is properly cited.

A coupled numerical model for nanorod-based suspension flow is constructed, and the convective heat transfer and resistance characteristics of the nanofluid duct flow are investigated. The numerical results are verified by experimental results and theoretical models. Most of nanorods are located randomly in the bulk fluid, while particles near the wall aligned with the flow direction. Friction factor of nanofluids with nanorods increases with higher particle volume concentration or aspect ratio, but the increment reduces when the Reynolds number gets larger. The relative Nusselt number is obtained to characterize the intensity of convective heat transfer. The results show that the Nusselt number of nanofluids increases when the particle volume concentration or aspect ratio becomes larger. Compared to increasing the aspect ratio of nanorods, increasing the particle volume concentration would be more effective on enhancing the convective heat transfer intensity in industrial applications although it will cause a slight increase of resistance.

\section{Introduction}

Nanofluids, which are fluids with nanosized particles suspended, are considered to be next-generation mediums for advanced heat transfer or cooling technology [1]. Since Choi put forward the concept firstly [2], the study of nanofluids has been intensively performed and reported in worldwide. Abnormal heat transfer characteristics were reported by experiments including the effective heat conductivity, natural and forced convection heat transfer, and boiling heat transfer of nanofluids [3-5]. Factors that can influence thermophysical properties have been discussed in many literatures, such as nanoparticle size and shape, material of particle and base fluid, particle loading, temperature, and so on, but quantitative understanding is still a lack on the topic $[1,3]$. Another hot topic is the flow resistance characteristic of nanofluids $[6,7]$. The pumping power needed for the bulk fluid did not increase dramatically as the particle loading grows in experiments [5], whereas the viscosity of nanofluids shows a marked increase after adding nanoparticles into base fluid $[1,6]$. The rheology of nanofluids also leads to the complexity on characterizing physical properties of bulk flow $[8,9]$.

Although many researchers have reviewed lots of measured experimental data and demonstrated that particle shape plays an important role on convective heat transfer characteristic of nanofluids [10, 11], few models can be found in literatures proposed for nanofluid flow containing nonspherical nanoparticles. Nanorods, as well as nanotubes, nanofibers, and nanobelts are used more and more for nanofluid preparation because of larger aspect ratio and surface area compared with spherical nanoparticles. Such kind of one-dimensional nanomaterials are available for heat transfer application with the development of nanoscale synthesis technology [12]. Xie et al. [13] first found the thermal conductivity of nanofluids with elongated nanoparticles is superior to that of spherical nanoparticles. Murshed et al. [14] measured enhanced thermal conductivity of water-based nanofluid containing $\mathrm{TiO}_{2}$ nanorods with the aspect ratio of 4 ; they showed that the results 
give $12 \%$ higher than that predicted by classical HamiltonCrosser model. Then, two models derived considering interfacial layer existence between particle and base fluid for the effective thermal conductivities of nanofluids with spherical and cylindrical nanoparticles [15]. Yang and Han [16] investigated the thermal conductivity of nanofluids dispersed by $\mathrm{Bi}_{2} \mathrm{Te}_{3}$ nanorod in perfluoro- $n$-hexane and oil. They found the thermal conductivities of nanofluids decrease with increasing temperature due to nanorod aspect ratio. The results measured by Zhang et al. [17, 18] employing carbon nanotubes (CNTs) agreed well with the predictions of the unit-cell model proposed by Yamada and Ota [19] by considering shape factor for cylindrical particles. By using the excluded volume concept, Koo et al. [20] constructed a model which can reproduce the nonlinear increase of the thermal conductivity for carbon nanotube and nanofiber suspensions. Timofeeva et al. [21] studied the particle shape effect on thermophysical properties of alumina-EG/ $\mathrm{H}_{2} \mathrm{O}$ suspensions and concluded that elongated particles and agglomerates resulted in higher viscosity at the same volume fraction due to structural limitation of rotational enhancements in effective thermal conductivities diminished by interfacial effects. Molecular dynamics (MD) simulation based on the model developed by Ghosh et al. [22] have shown that cylindrical nanoparticles pick up thermal energy much faster than spherical nanoparticles during a collision with a block-shaped heat source [23]. Comparative experimental results reported by $\mathrm{Yu}$ et al. [10] indicated that convective heat transfer characteristic of nanofluids containing nonspherical nanoparticles is affected significantly by the shear-induced alignment and orientational motion of particles. By considering the thickness and thermal conductivity of the interfacial nanolayer, Jiang et al. [24] proposed a model which provides good predictions for the effective thermal conductivity of CNTbased nanofluids. Other existing models are summarized by Yang et al. [11], and three similar theoretical models are proposed for nanofluids with finite cylindrical particles by anisotropy analysis [25-27]. All of the three models give minimal relative errors to experimental results compared to other models. Lin et al. $[7,28]$ took numerical simulation on the phaolefins-based nanofluids fluid flow containing cylindrical $\mathrm{Al}_{2} \mathrm{O}_{3}$ nanoparticles in laminar and turbulent pipes, respectively. The results show that the friction factor of flow decreases when the Reynolds number and particle aspect ratio become larger. The Nusselt number of convective heat transfer increases with the increase of Reynolds number, particle aspect ratio, and volume concentration. By comparing the defined performance evaluation criterion (PEC), nanofluids containing rod-like nanoparticles with large aspect ratio and a suitable particle volume concentration are more effective for convective heat transfer process at higher Reynolds number. A coupled numerical model is constructed by Yuan et al. [29] to simulate convective heat transfer and resistance characteristics of $\mathrm{TiO}_{2}$ /water nanofluids with cylindrical particles in laminar channel flow. The results show that the Nusselt number of nanofluid flow related to the Reynolds number, axial length, Prandtl number, and particle volume concentration and a fitted formula is proposed to predict the Nusselt number of nanofluid flow containing cylindrical nanoparticles.
As summarized above, high potential of rod-like nanoparticles in enhancing thermal conductivity of nanofluids has got more attention by researchers. Related reports focus mostly on viscosity and thermal conductivity properties, while progress made on convective heat transfer characteristics is still highly needed. In addition, lack of numerical modelling on nanofluid flow brings an obstacle to industrial applications. The objective of this work is to develop a coupled model for nanorod-based suspension flow and investigate the convective heat transfer and resistance characteristics of the nanofluid duct flow numerically. The orientation and movement of nanorod are considered, and key factors that can influence the friction factor and Nusselt number of the fluid flow are discussed in sequence.

\section{Model for Nanorod-Based Nanofluids}

2.1. Governing Equations. The duct flow for nanofluid is considered as impressible, and the governing equations include the continuity equation, modified Navier-Stokes equation with the additional term of nanorods, and the energy conservation equation $[28,30,31]$ :

$$
\begin{aligned}
\frac{\partial u_{i}}{\partial x_{i}}= & 0, \\
\frac{D u_{i}}{D t}= & -\frac{1}{\rho_{\mathrm{nf}}} \frac{\partial p}{\partial x_{i}}+\frac{\mu}{\rho_{\mathrm{nf}}} \frac{\partial^{2} u_{i}}{\partial^{2} x_{j}} \\
& +\frac{\mu_{a}}{\rho_{\mathrm{nf}}} \frac{\partial}{\partial x_{j}}\left[a_{i j k l} \varepsilon_{k l}-\frac{1}{3}\left(I_{i j} a_{k l}\right) \varepsilon_{k l}\right], \\
\frac{D T}{D x_{j}}= & \left(C_{\mathrm{nf}}+C_{T}\right) \frac{\partial^{2} T}{\partial x_{j}^{2}},
\end{aligned}
$$

where $u_{i}, p, \rho_{\mathrm{nf}}$, and $T$ are the nanofluid velocity, pressure, density, and temperature, respectively; $\mu$ is the dynamic viscosity of the pure fluid; $\mu_{\mathrm{a}}$ is the apparent viscosity; $\bar{a}_{k l}$ and $\bar{a}_{i j k l}$ are the mean second- and fourth-order tensors of particle orientation, respectively; $\varepsilon_{i j}=\left(\partial u_{i} / \partial x_{j}+\partial u_{j} / \partial x_{i}\right) / 2$ is the rate-of-strain tensor; and $C_{\mathrm{nf}}$ is the thermal diffusivity coefficient of the nanofluid, $C_{T}=C_{\mu} k^{2} / \varepsilon \operatorname{Pr}_{T}$ ( $k$ is the turbulent kinetic energy, $\varepsilon$ is the turbulent dissipation rate, $C_{\mu}=0.09$, and turbulent Prandtl number, $\operatorname{Pr}_{\mathrm{T}}=0.9$ ) is the eddy thermal diffusivity coefficient.

Substituting instantaneous velocity, pressure, temperature, rate-of-strain tensor, and tensors of particle orientation which consist of mean and fluctuation part in (1-3) and averaging, we have

$$
\begin{aligned}
\frac{\partial U_{i}}{\partial x_{i}}= & 0 \\
\frac{D U_{i}}{D t}= & -\frac{1}{\rho_{\mathrm{nf}}} \frac{\partial P}{\partial x_{i}}+\frac{\mu}{\rho_{\mathrm{nf}}} \frac{\partial^{2} U_{i}}{\partial^{2} x_{j}}-\frac{\partial \overline{u_{i}^{\prime} u_{j}^{\prime}}}{\partial x_{j}} \\
& +\frac{\mu_{a}}{\rho_{\mathrm{nf}}} \frac{\partial}{\partial x_{j}}\left[\bar{a}_{i j k l} \bar{\varepsilon}_{k l}-\frac{1}{3}\left(I_{i j} \bar{a}_{k l}\right) \bar{\varepsilon}_{k l}\right], \\
\frac{D \bar{T}}{D t}= & \left(C_{\mathrm{nf}}+C_{T}\right) \frac{\partial^{2} \bar{T}}{\partial x_{j}^{2}},
\end{aligned}
$$


where $U_{i}, P$, and $\bar{T}$ are the mean nanofluid velocity, pressure, and temperature, respectively, $u_{i}^{\prime}$ is the fluctuation flow velocity, and $\bar{\varepsilon}_{k l}$ is the mean rate-of-strain tensor.

In (5), the nanofluid density is

$$
\rho_{\mathrm{nf}}=\left(1-\phi_{\mathrm{e}}\right) \rho_{\mathrm{f}}+\phi_{\mathrm{e}} \rho_{\mathrm{p}},
$$

where the subscripts "f " and "p" stand for pure fluid and nanoparticles, respectively, and $\phi_{\mathrm{e}}$ is the effective particle volume concentration, which is used for replacing the nominal particle volume fraction $\phi$, in consideration of the particle aggregation:

$$
\phi_{\mathrm{e}}=\phi\left(s / s_{e}\right)^{3-f_{i}},
$$

where $s$ and $s_{e}$ are the effective size of aggregates and primary particle size, respectively, and $f_{i}$ is the fractal index and varies from 1.5 to 2.45 for those of rod-like nanoparticles. Yu et al. [10] gives the value as: $s / s_{e}=1.48$ and $f_{i}=1.95$ according to experimental data.

Batchelor's theory was extended by Mackaplow and Shaqfeh [32] to account for two-body interactions and gave the apparent viscosity in (5):

$\mu_{a}=\frac{1}{6} \pi n l^{3} \mu\left\{\frac{1}{\ln (2 r)}\left[\frac{\ln (2 r)+0.640}{\ln (2 r)-1.5}+\frac{1.659}{\ln (2 r)^{2}}\right]+\frac{0.1515 n l^{3}}{\ln (2 r)^{3}}\right\}$,

where $n$ and $l$ are the number density and half length of nanorods, respectively, $\mu$ is the dynamic viscosity of the pure fluid, and $r$ is the particle aspect ratio, which is the ratio of length of diameter.

The thermal diffusivity coefficient in $(6)$ is $[33,34]$

$$
C_{\mathrm{nf}}=\frac{k_{\mathrm{nf}}}{\left(\rho C_{p}\right)_{\mathrm{nf}}},
$$

where the heat capacitance of the nanofluid is given by

$$
\left(\rho C_{\mathrm{p}}\right)_{\mathrm{nf}}=\left(1-\phi_{\mathrm{e}}\right)\left(\rho C_{\mathrm{p}}\right)_{\mathrm{f}}+\phi_{\mathrm{e}}\left(\rho C_{\mathrm{p}}\right)_{\mathrm{p}}
$$

In (10), the classical Hamilton-Crosser model is employed for predicting thermal conductivity of nanorodbased nanofluid as follows:

$$
k_{\mathrm{nf}}=k_{f}\left[\frac{k_{p}+k_{f}(K-1)+(K-1) \phi_{e}\left(k_{p}-k_{f}\right)}{k_{p}+k_{f}(K-1)-\phi_{e}\left(k_{s}-k_{f}\right)}\right],
$$

in which $k_{\mathrm{f}}$ and $k_{\mathrm{p}}$ are the thermal conductivity of pure fluid and nanoparticles, respectively, and $K$ is the shape factor given by $K=3 / \gamma$, where $\gamma$ is the particle sphericity, defined as the ratio of the surface area of a sphere with the same volume as that of the particle and the surface area of the particle. The model predicts well the thermal conductivity of nanofluid with $\mathrm{ZnO}$ nanoparticles with experimental data by Ferrouillat et al. [35] in rod-like shape.

The Reynolds stress tensor $-\rho \overline{u_{i}^{\prime} u_{j}^{\prime}}$ in (5) is

$$
-\rho_{\mathrm{nf}} \overline{u_{i}^{\prime} u_{j}^{\prime}}=2 \mu_{T}\left(\frac{\partial U_{i}}{\partial x_{j}}+\frac{\partial U_{j}}{\partial x_{i}}\right)-\frac{2}{3} \rho_{\mathrm{nf}} k \delta_{i j} \text {, }
$$

where eddy viscosity $\mu_{T}=0.09 \rho_{\mathrm{nf}} k^{2} / \varepsilon$. For solving (5), the $k$-equation and $\varepsilon$-equation for turbulent flow are given by

$$
\begin{aligned}
\rho_{\mathrm{nf}} U_{j} \frac{\partial k}{\partial x_{j}}= & -\rho_{\mathrm{nf}} \overline{u_{i}^{\prime} u_{j}^{\prime}} \frac{\partial U_{i}}{\partial x_{j}}-\rho_{\mathrm{nf}} \varepsilon+\frac{\partial}{\partial x_{j}}\left[\left(\mu_{a}+\frac{\mu_{T}}{\sigma_{k}}\right) \frac{\partial k}{\partial x_{j}}\right], \\
\rho_{\mathrm{nf}} U_{j} \frac{\partial \varepsilon}{\partial x_{j}}= & -C_{1} \frac{\varepsilon}{k} \rho_{\mathrm{nf}} \overline{u_{i}^{\prime} u_{j}^{\prime}} \frac{\partial U_{i}}{\partial x_{j}}-C_{2} \rho_{\mathrm{nf}} \frac{\varepsilon^{2}}{k} \\
& +\frac{\partial}{\partial x_{j}}\left[\left(\mu_{a}+\frac{\mu_{T}}{\sigma_{\varepsilon}}\right) \frac{\partial \varepsilon}{\partial x_{j}}\right],
\end{aligned}
$$

where $C_{1}=1.44, C_{2}=1.92, \sigma_{k}=1.0$, and $\sigma_{\varepsilon}=1.3$.

2.2. Nanoparticle Orientation Distribution. The mean second- and fourth-order tensors of particle orientation in (5) describe the orientation distribution of nanoparticle population in flow field; they can be calculated by Advani and Tucker [36] as

$$
\begin{aligned}
\bar{a}_{i j} & =\oint p_{i} p_{j} \overline{\psi(\mathbf{p})} d \mathbf{p}, \\
\bar{a}_{i j k l} & =\oint p_{i} p_{j} p_{k} p_{l} \overline{\psi(\mathbf{p})} d \mathbf{p},
\end{aligned}
$$

where $p_{i}$ is a unit vector parallel to the particle's axis and $\overline{\psi(\mathbf{p})}$ is the probability density function for particle orientation at any position with $\mathbf{p}$ being the orientation vector. The governing equation of $\psi(\mathbf{p})$ is the modified FokkerPlanck equation considering the rotary effect caused by Brownian movement $[37,38]$ :

$$
\frac{\partial \psi}{\partial t}+u_{j} \frac{\partial \psi}{\partial x_{j}}=D_{\mathrm{rB}} \frac{\partial^{2} \psi}{\partial p_{j}^{2}}-\frac{\partial\left(\psi \dot{p}_{j}\right)}{\partial p_{j}},
$$

where $D_{\mathrm{rB}}$ is the Brownian rotary diffusion coefficient, $\partial / \partial p_{j}$ is the gradient operator projected onto the surface of the unit sphere, and $\dot{p}_{j}$ is the particle angular velocity. Cintra and Tucker [39] expressed $\dot{p}_{i}$ as

$$
\dot{p}_{i}=-\omega_{i j} p_{j}+\lambda \varepsilon_{i j} p_{j}-\lambda \varepsilon_{k l} p_{k} p_{l} p_{i}-\frac{D_{\mathrm{rI}}}{\psi} \frac{\partial \psi}{\partial p_{i}},
$$

where $\omega_{i j}=\left(\partial u_{j} / \partial x_{i}-\partial u_{i} / \partial x_{j}\right) / 2$ is the vorticity tensor, $\lambda=\left(r^{2}-1\right) /\left(r^{2}+1\right)$. The last term in (18) is introduced to model the behavior at higher concentrations, in which $D_{\mathrm{rI}}$ is a rotary diffusion coefficient resulting from particle-particle interactions. Folgar and Tucker [40] suggested that $D_{\mathrm{rI}}$ is isotropic and given by $C_{I} \sqrt{2 \varepsilon_{i j} \varepsilon_{j i}}$, where the interaction coefficient $C_{I}$ is fixed to 0.01 .

For cylindrical particles, the Brownian rotary diffusion coefficient in (17) is

$$
D_{\mathrm{rB}}=\frac{k_{b} T}{\sqrt{\gamma_{\mathrm{rL}}^{2}+\gamma_{\mathrm{rS}}^{2}}} .
$$

With aspect ratio $r$, the rotational friction coefficients around long axis and short axis are [41, 42]. 


$$
\begin{aligned}
& \gamma_{\mathrm{rL}}=\frac{3.84 \pi \mu L^{3}\left(1+\delta_{\mathrm{rL}}\right)}{r^{2}}, \\
& \gamma_{\mathrm{rS}}=\frac{\pi \mu L^{3}}{3\left(\ln r+\delta_{\mathrm{rS}}\right)},
\end{aligned}
$$

with

$$
\begin{aligned}
& \delta_{\mathrm{rL}}=\frac{0.677}{r}-\frac{0.183}{r^{2}}, \\
& \delta_{\mathrm{rS}}=-0.662+\frac{0.917}{r}-\frac{0.05}{r^{2}} .
\end{aligned}
$$

Substituting instantaneous $\psi$ and $\dot{p}_{j}$ which consist of mean and fluctuation part in (17) and averaging, we have the mean equation of probability density functions for the particle orientation:

$$
\begin{aligned}
& \frac{\partial \bar{\psi}}{\partial t}+\bar{u}_{j} \frac{\partial \bar{\psi}}{\partial x_{j}}-D_{\mathrm{rB}} \frac{\partial^{2} \bar{\psi}}{\partial p_{j}^{2}}-\bar{\omega}_{j i} p_{i} \frac{\partial \bar{\psi}}{\partial p_{j}}+K \bar{\varepsilon}_{j i} p_{i} \frac{\partial \bar{\psi}}{\partial p_{j}} \\
& -K \bar{\varepsilon}_{k l} p_{k} p_{l} p_{j} \frac{\partial \bar{\psi}}{\partial p_{j}}-K \bar{\varepsilon}_{k l} \bar{\psi} p_{k} p_{l}-D_{\mathrm{rI}} \frac{\partial^{2} \bar{\psi}}{\partial p_{j}^{2}}=\alpha_{\psi p} \frac{\partial^{2} \bar{\psi}}{\partial p_{j}^{2}}
\end{aligned}
$$

where $\alpha_{\psi x}=1.3\left(5 k^{2} \nu / 3 \varepsilon\right)^{1 / 2}$ and $\alpha_{\psi p}=0.7(4 \varepsilon / 15 \nu)^{1 / 2}$ are the dispersion coefficients of linear and angular displacement in which $v$ is the kinetic viscosity of the pure fluid [43], which appear based on the dispersion mechanism:

$$
\begin{aligned}
& -\overline{\psi^{\prime} u_{j}^{\prime}}=\alpha_{\psi x} \frac{\partial \bar{\psi}}{\partial x_{j}}, \\
& -\overline{\psi^{\prime} \dot{p}_{j}^{\prime}}=\alpha_{\psi p} \frac{\partial \bar{\psi}}{\partial p_{j}} .
\end{aligned}
$$

Integral on the angle after multiplying component of unit vector $p_{i},(22)$ is transformed to the equation about $\bar{a}_{i j}$ :

$$
\begin{aligned}
\frac{D \bar{a}_{i j}}{D t}= & -\left(\bar{\omega}_{i k} \bar{a}_{k j}-\bar{a}_{i k} \bar{\omega}_{k j}\right)+K\left(\bar{\varepsilon}_{i k} \bar{a}_{k j}+\bar{a}_{i k} \bar{\varepsilon}_{k j}-2 \bar{\varepsilon}_{k l} \bar{a}_{i j k l}\right) \\
& +2\left(\alpha_{\psi p}+D_{\mathrm{rI}}+D_{\mathrm{rB}}\right)\left(\delta_{i j}-\alpha \bar{a}_{i j}\right) .
\end{aligned}
$$

The mean fourth-order particle orientation tensor needs to be closured by model. Considering the shearing-stretch feature of duct flow, the orthotropic closure model [44] is fit for (24):

$$
\begin{aligned}
\bar{a}_{i j k l}= & \frac{\operatorname{det}\left(\bar{a}_{i j}\right)}{6}\left(\delta_{i j} \delta_{k l}+\delta_{i k} \delta_{j l}+\delta_{i l} \delta_{j k}\right) \\
& +\frac{1}{3}\left(\bar{a}_{i j} \bar{a}_{k l}+\bar{a}_{i k} \bar{a}_{j l}+\bar{a}_{i l} \bar{a}_{j k}\right),
\end{aligned}
$$

in which $\operatorname{det}\left(a_{i j}\right)$ is the determinant of second-order tensor $\mathbf{a}_{2}$.

2.3. General Dynamics Equation for Rod-Like Nanoparticles. The particle volume concentration $\phi$ in (7), (11), and (12) is considered distribution inhomogeneity in the flow. In order to obtain the distribution of $\phi$ in the flow, it is necessary to solve the general dynamics equation for nanorods:

$$
\frac{D n(v)}{D t}-\frac{\partial}{\partial x_{j}} D_{\mathrm{tB}} \frac{\partial n(v)}{\partial x_{j}}=0 .
$$

Based on the Reynolds average, the above equation is transformed to

$$
\frac{D \bar{n}(v)}{D t}-\frac{\partial}{\partial x_{j}} D_{\mathrm{tB}} \frac{\partial \bar{n}(v)}{\partial x_{j}}+\frac{\partial \overline{n^{\prime}(v) u_{j}^{\prime}}}{\partial x_{j}}=0
$$

where $\bar{n}(v)$ is the mean particle volume distribution function based on the volume and the last term on the left-hand side represents the change in $\bar{n}(v)$ resulting from turbulent diffusion and is usually assumed to be [45]:

$$
\overline{n^{\prime}(v) u_{j}^{\prime}}=-\varepsilon_{i} \frac{\partial \bar{n}(v)}{\partial x_{j}},
$$

where $\varepsilon_{i}$ is the eddy diffusion coefficient which is a function of position and $\varepsilon_{i}=v_{\mathrm{t}} / \mathrm{Sc}_{\mathrm{t}}$, where $v_{\mathrm{t}}$ and $\mathrm{Sc}_{\mathrm{t}}$ are the turbulent viscosity and the Schmidt number, respectively.

$D_{\mathrm{tB}}$ in (26) is the Brownian translational diffusion coefficient for rod-like nanoparticles:

$$
D_{\mathrm{tB}}=\frac{k_{b} T}{\sqrt{\gamma_{\mathrm{tL}}^{2}+\gamma_{\mathrm{tS}}^{2}}},
$$

and the translational friction coefficients parallel and perpendicular to the long axis are $[41,42]$

$$
\begin{aligned}
& \gamma_{\mathrm{tL}}=\frac{2 \pi \mu L}{\ln r+\delta_{\mathrm{tL}}}, \\
& \gamma_{\mathrm{rS}}=\frac{4 \pi \mu L}{\ln r+\delta_{\mathrm{tS}}},
\end{aligned}
$$

with

$$
\begin{aligned}
& \delta_{\mathrm{tL}}=-0.207+\frac{0.980}{r}-\frac{0.133}{r^{2}} \\
& \delta_{\mathrm{rS}}=0.839+\frac{0.185}{r}+\frac{0.233}{r^{2}}
\end{aligned}
$$

Substituting (29) in (27), we have averaged general dynamics equation for nanoparticles. With the moment transformation which involves multiplying the equation by $v^{k}$ and then integrating over the entire size regime, the equation for rod-like nanoparticles finally becomes

$$
\frac{\partial m_{k}}{\partial t}+\bar{u}_{j} \frac{\partial m_{k}}{\partial x_{j}}-\frac{\partial}{\partial x_{j}}\left[\left(\frac{k_{b} T}{\sqrt{\gamma_{\mathrm{tL}}^{2}+\gamma_{\mathrm{tS}}^{2}}}+\varepsilon_{i}\right) \frac{\partial m_{k}}{\partial x_{j}}\right]=0,
$$

where the zero moment and the first-order moment

$$
\begin{aligned}
& M_{0}=\int_{0}^{\infty} \bar{n}(v) \mathrm{dv}=N, \\
& M_{1}=\int_{0}^{\infty} v \bar{n}(v) \mathrm{dv}=V,
\end{aligned}
$$

describe the total particle number density and volume of nanoparticles at a given point. 


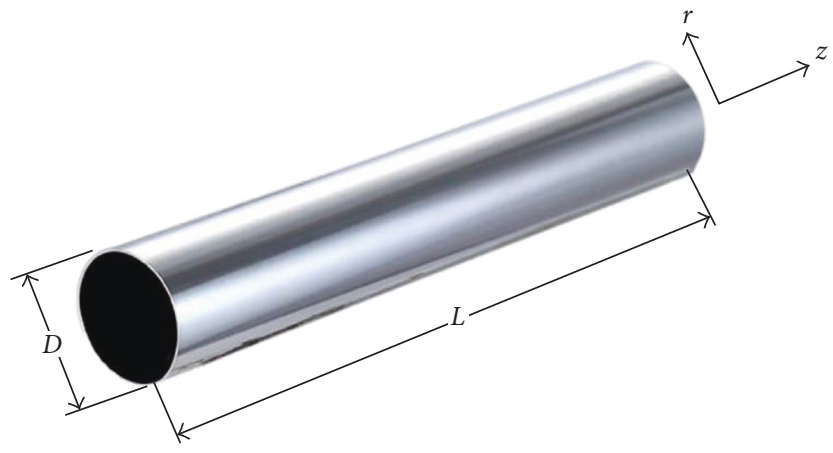

Figure 1: Schematic of a nanofluid pipe flow.

TABLE 1: Thermophysical properties of materials.

\begin{tabular}{lcc}
\hline Thermophysical properties & $\begin{array}{c}\mathrm{H}_{2} \mathrm{O} \\
\text { (base fluid) }\end{array}$ & $\begin{array}{c}\mathrm{ZnO} \\
\text { (nanorods) }\end{array}$ \\
\hline$\rho\left(\mathrm{kg} \cdot \mathrm{m}^{-3}\right)$ & 998.2 & 5606 \\
$C_{p}\left(\mathrm{~J} \cdot \mathrm{kg}^{-1} \cdot \mathrm{K}^{-1}\right)$ & 4182 & 5200 \\
$k\left(\mathrm{~W} \cdot \mathrm{m}^{-1} \cdot \mathrm{K}^{-1}\right)$ & 0.62 & 60 \\
\hline
\end{tabular}

\section{Numerical Method}

Schematic of a turbulent duct flow containing nanorodbased nanofluid is shown in Figure 1. The length and diameter of the channel are $L$ and $D$, respectively.

The boundary conditions are given as follows: inlet: $U_{x}=U_{0}, U_{y}=0, T=T_{\mathrm{in}}, m_{1}=m_{10}$; outlet: $\partial U_{x} / \partial x=0, U_{y}=0$, $\partial T / \partial x=0, \partial m_{0} / \partial x=0$; and wall: $U_{x}=U_{y}=0, m_{1}=0$, $\partial m_{0} / \partial x=0$. Notably, numerical simulations performed here are forced convection process with uniform wall temperature, other than those with uniform wall heat flux.

The ratio of channel length to height is $L / D=125$. The nanofluid is a mixture of water and rigid $\mathrm{ZnO}$ nanorods. Thermophysical properties of water and $\mathrm{ZnO}$ are presented in Table 1. The temperatures of inlet nanofluid and wall are $T_{\text {in }}=293 \mathrm{~K}$ and $T_{w}=323 \mathrm{~K}$, respectively. Reynolds number of the fluid flow is defined as $\operatorname{Re}=u_{0} D / v_{\text {nf }}$ ranging from 2500 to 15,000 . The nominal particle volume fraction $\phi$ for nanofluids is $0.4 \%, 0.93 \%$, and $1.3 \%$, and aspect ratio $r$ is 8,12 and 16 for comparing with experimental results in Ferrouillat et al. [35].

The SIMPLEC algorithm is employed for solving coupled continuity equation and moment equation, where the convection item and diffusion item are discreted using QUICK scheme and second-order center difference, respectively. No slip condition is set for wall boundary condition.

\section{Results and Discussion}

4.1. Calculation Verification. To validate the model and code for nanofluid flow, classical models and experimental results are used for comparing the numerical results of friction factor and the Nusselt number of duct fluid flow in Figures 2 and 3. The definition of $f$ and $\mathrm{Nu}$ is as follows:

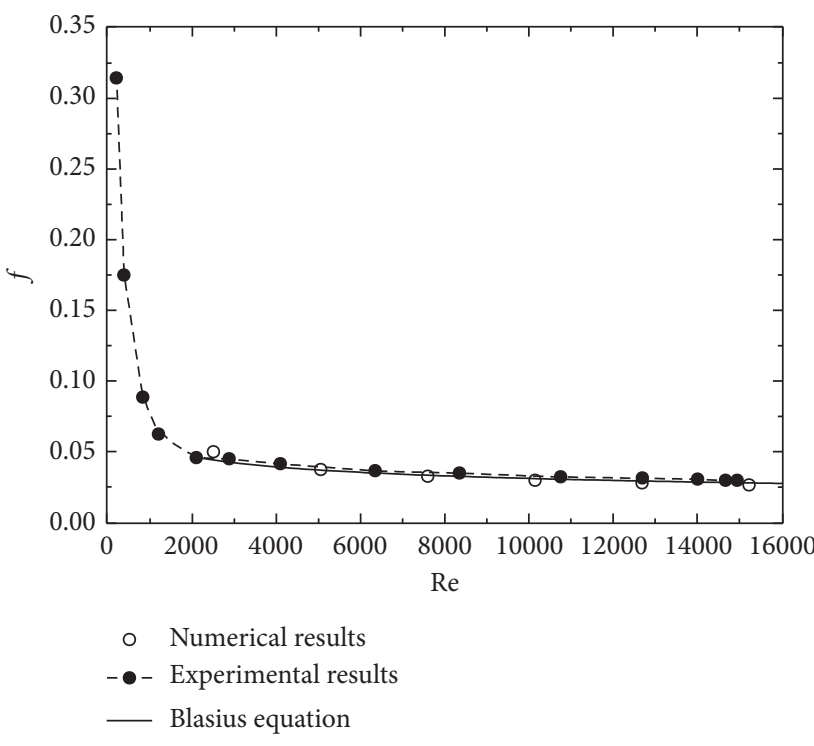

Figure 2: Comparison of friction factor of nanofluids.

$$
\begin{aligned}
f & =\frac{\Delta p}{\rho_{\mathrm{nf}}(L / D)\left(U_{\mathrm{av}}^{2} / 2\right)}, \\
\mathrm{Nu} & =\frac{h D}{k_{\mathrm{nf}}}=\frac{-\left.k_{\mathrm{nf}}(\partial T / \partial r)\right|_{r= \pm D / 2}}{k_{\mathrm{nf}}}=-\left.\frac{\partial T}{\partial r}\right|_{r= \pm D / 2},
\end{aligned}
$$

where $\Delta p$ is the pressure drop, $U_{\mathrm{av}}$ is the average velocity of the fluid in the flow direction, and $h$ is the heat transfer coefficient.

Figure 2 shows the comparison of friction factor of nanofluids varying with the Reynolds number. The solid points in figure are measured by Ferrouillat et al. [35], and the solid curve is the Blasius equation which is $f=0.316 \mathrm{Re}^{-0.25}$ for turbulent duct flow. The numerical results are drawn by hollow points and are obtained by setting the nanorod aspect ratio as 8 , because the aspect ratio of nanoparticles in reference experiments are about 6 10. From the figure, the numerical results show agreement with the experimental results and the curve of Blasius equation. In turbulence regime, the friction factor of nanofluid flow drops slowly when the Reynolds number grows.

Comparison of calculated Nusselt number of nanofluids with Dittus-Boelter relation is shown in Figure 3. The classical Dittus-Boelter relation is commonly used for predicting the Nusselt number in fully developed turbulent duct flow, which is $\mathrm{Nu}=0.023 \mathrm{Re}^{0.8} \mathrm{Pr}^{0.4}$. The difference of Nusselt number predicted by current model and D-B relation is within about $10 \%$, and the difference declines in high $\mathrm{Nu}$ region.

4.2. Orientation Distribution of Nanorods. The orientation of nanorods in fluid makes an effect on nanofluid fluid flow. The orientation distribution of nanorods population can be described by the mean second-order orientation tensor of particle. The components of $\bar{a}_{i j}$ in $z$ direction are shown in Figure 4 , in which the volume concentration and aspect ratio 


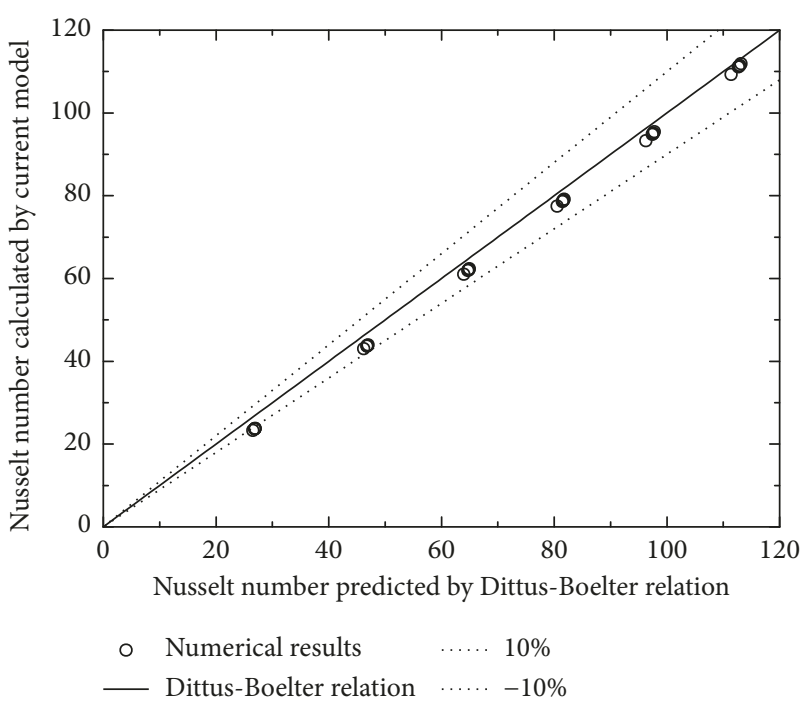

Figure 3: Comparison of calculated Nusselt number of nanofluids with Dittus-Boelter relation.

are set as $\phi=1.4 \%$ and $r=16$, respectively. It can be seen from the figure that the distributions of components of $a_{i i}$ coincide with each other, and the median distribution of $\bar{a}_{i i}$ which along the axial direction is $1 / \pi$. The value of $\bar{a}_{i i}$ near the wall is larger that that of central area, which means that the nanorods align with the flow direction due to the existence of wall. The orientation state of nanorods can hardly keep in $y-z$ component because $\bar{a}_{23}$ is always zero in $z$ direction. But $\bar{a}_{13}$ is not zero near the wall, which illustrates the wall effect on orientation state of nanorods.

4.3. Friction Factors. The friction factor and pressure drop of the duct flow are of importance to the practical applications. Figure 5 shows the friction factors of nanofluids at different volume concentrations as a function of the Reynolds number. The aspect ratio of nanorods in experiments and simulations are set as $r=8$. All the friction factors of nanofluid flow are higher than those of base fluid and decrease when the Reynolds number grows higher. The friction factor increases slightly with the increase of particle volume concentration, and the difference diminishes when the Reynolds number becomes larger.

In Figure 6, the friction factor as a function of the Reynolds number by different aspect ratio is given, and the volume concentration $\phi$ is $0.93 \%$. As can be seen in figure, the shape of nanoparticles takes effect on friction factor of nanofluid fluid flow. Nanorods with larger aspect ratio bring more flow resistance when the Reynolds number is the same. When the Reynolds number gets larger, the friction factor of nanofluid approaches to that of base fluid, which means the additional pumping power for nanofluid is not significant in high Reynolds number region.

4.4. Convective Heat Transfer. The Nusselt number describes the ratio of convective heat transfer across the wall. The relative Nusselt number is defined here as the ratio of the

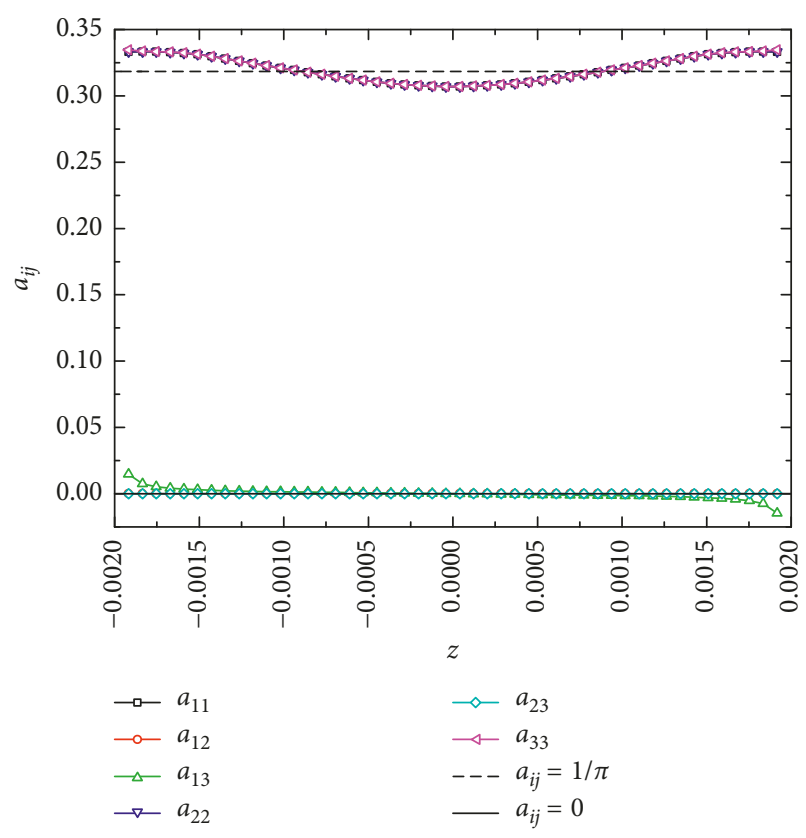

Figure 4: Components of $a_{i j}$ in $z$ direction $(\phi=1.4 \%, r=16)$.

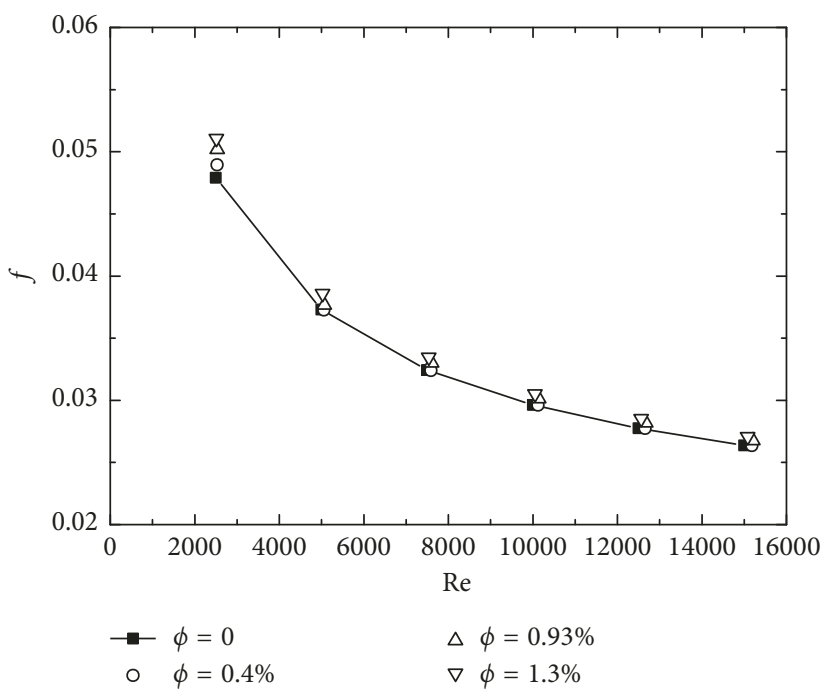

FIgURE 5: Friction factor as a function of the Reynolds number by different volume concentrations $(r=8)$.

Nusselt number of nanofluids to that of base fluid, that is, $\mathrm{Nu}_{\mathrm{nf}} / \mathrm{Nu}_{\mathrm{f}}$. Figures 7 and 8 show the calculated and experimental results of $\mathrm{Nu}_{\mathrm{nf}} / \mathrm{Nu}_{\mathrm{f}}$ as a function of the Reynolds number with different particle aspect ratios or different volume concentrations. The solid points give the measured $\mathrm{Nu}$ with particle volume concentration as $0.93 \%$ and reveal that the Nusselt number of nanofluid is smaller than that of base fluid when $\mathrm{Re}$ is less than 7000 . The overall experimental data indicate that $\mathrm{Nu}_{\mathrm{nf}} / \mathrm{Nu}_{\mathrm{f}}$ grows as the Reynolds number gets larger except the point when Re is about 2100 . The calculated $\mathrm{Nu}_{\mathrm{nf}} / \mathrm{Nu}_{\mathrm{f}}$ by model in this paper is larger than that of experiments when the volume concentration is set as $0.93 \%$. In addition, all the numerical results are greater 


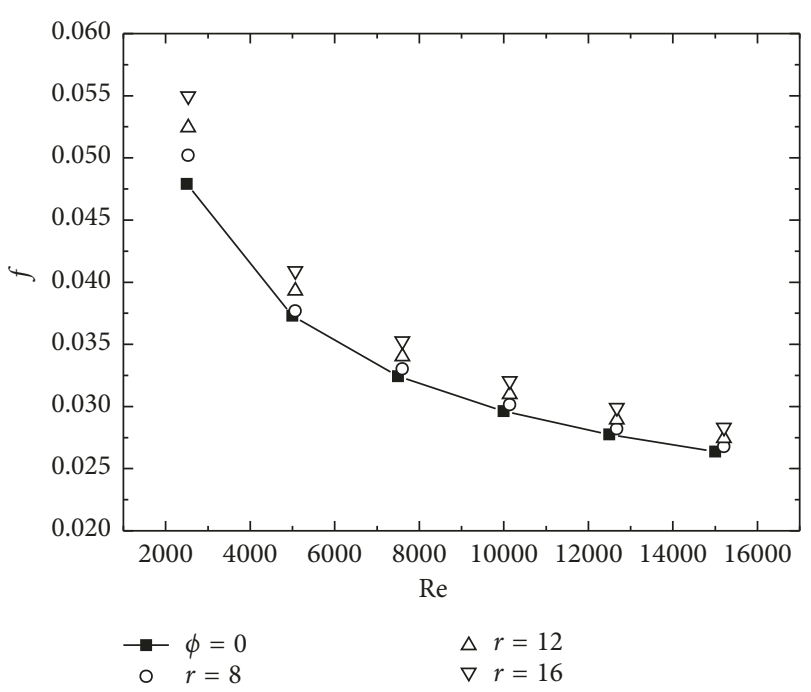

FIGURE 6: Friction factor as a function of the Reynolds number by different aspect ratios $(\phi=0.93 \%)$.

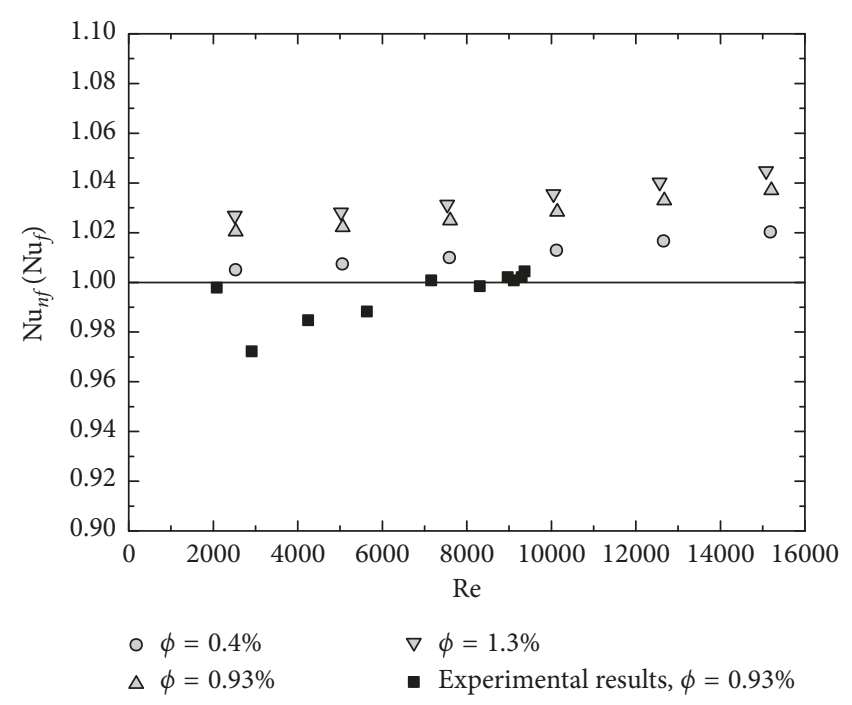

Figure 7: $\mathrm{Nu}_{\mathrm{nf}} / \mathrm{Nu}_{\mathrm{f}}$ as a function of the Reynolds number by different volume concentrations $(r=8)$.

than 1 , which means the convective heat transfer of nanofluid is better than base fluid at the same Reynolds number. $T$ The $\mathrm{Nu}_{\mathrm{nf}} / \mathrm{Nu}_{\mathrm{f}}$ is monotone increasing as a function of the Reynolds number, no matter the particle volume concentration or aspect ratio varies in figures. In Figure 7, the relative Nusselt number increases as the particle volume concentration gets larger. Similarly, in Figure 8, the relative Nusselt number increases as the particle aspect ratio gets larger. Both the particle loading and particle shape influence the convective heat transfer characteristic obviously. Compared to increasing the aspect ratio of nanorods, increasing the particle volume concentration would be more effective on enhancing the convective heat transfer intensity in industrial applications although it will cause a slight increase of resistance.

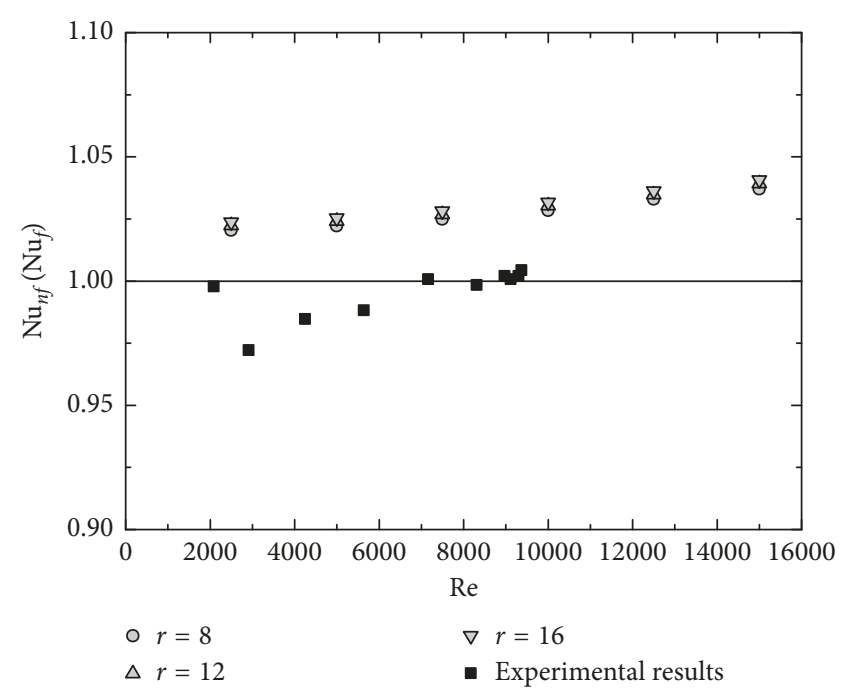

FIgURE 8: $\mathrm{Nu}_{\mathrm{nf}} / \mathrm{Nu}_{\mathrm{f}}$ as a function of the Reynolds number by different aspect ratios $(\phi=0.93 \%)$.

\section{Conclusions}

The convective heat transfer and resistance characteristics of the nanofluid duct flow are investigated based on a coupled numerical model for nanorod-based suspension flow. The numerical results are verified by experimental results and theoretical models. Most of nanorods are located randomly in the bulk fluid, while particles near the wall aligned with the flow direction. Friction factor of nanofluids with nanorods increases with higher particle volume concentration or aspect ratio, but the increment reduces when the Reynolds number getting larger. The relative Nusselt number is obtained to characterize the intensity of convective heat transfer. The results show that the Nusselt number of nanofluids increases when the particle volume concentration or aspect ratio becomes larger. Compared to increasing the aspect ratio of nanorods, increasing the particle volume concentration would be more effective on enhancing the convective heat transfer intensity in industrial applications although it will cause a slight increase of resistance.

\section{Conflicts of Interest}

The authors declare that they have no conflicts of interest.

\section{Acknowledgments}

This work is supported by the National Natural Science Foundation of China (no. 11632016) and Jiangsu Key Laboratory of Green Process Equipment (GPE201705).

\section{References}

[1] S. S. Murshed and P. Estellé, "State of the art review on viscosity of nanofluids," Renewable and Sustainable Energy Reviews, vol. 76, pp. 1134-1152, 2017. 
[2] S. Choi and J. Eastman, "Enhancing thermal conductivity of fluids with nanoparticles," ASME-Publications-Fed, vol. 231, pp. 99-106, 1995.

[3] L. Yang and K. Du, "A comprehensive review on heat transfer characteristics of $\mathrm{TiO}_{2}$ nanofluids," International Journal of Heat and Mass Transfer, vol. 108, pp. 11-31, 2017.

[4] M. Bahiraei, "Particle migration in nanofluids: a critical review," International Journal of Thermal Sciences, vol. 109, pp. 90-113, 2016.

[5] M. Islam, B. Shabani, G. Rosengarten, and J. Andrews, "The potential of using nanofluids in PEM fuel cell cooling systems: a review," Renewable and Sustainable Energy Reviews, vol. 48, pp. 523-539, 2015.

[6] R. Saxena, D. Gangacharyulu, and V. K. Bulasara, "Heat transfer and pressure drop characteristics of dilute alumina-water nanofluids in a pipe at different power inputs," Heat Transfer Engineering, vol. 37, no. 18, pp. 1554-1565, 2016.

[7] J. Lin, Y. Xia, and X. Ku, "Friction factor and heat transfer of nanofluids containing cylindrical nanoparticles in laminar pipe flow," Journal of Applied Physics, vol. 116, no. 13, p. 133513, 2014.

[8] S. Kim, C. Kim, W.-H. Lee, and S.-R. Park, "Rheological properties of alumina nanofluids and their implication to the heat transfer enhancement mechanism," Journal of Applied Physics, vol. 110, no. 3, p. 034316, 2011.

[9] H. Chen, Y. Ding, and C. Tan, "Rheological behaviour of nanofluids," New Journal of Physics, vol. 9, no. 10, p. 367, 2007.

[10] L. Yu, D. Liu, and F. Botz, "Laminar convective heat transfer of alumina-polyalphaolefin nanofluids containing spherical and non-spherical nanoparticles," Experimental Thermal and Fluid Science, vol. 37, pp. 72-83, 2012.

[11] L. Yang, X. Chen, M. Xu, and K. Du, "Roles of surfactants and particle shape in the enhanced thermal conductivity of $\mathrm{TiO}_{2}$ nanofluids," AIP Advances, vol. 6, no. 9, p. 095104, 2016.

[12] G. Cao and Y. Wang, Nanostructures and Nanomaterials: Synthesis, Properties and Applications, World Scientific, London, UK, 2011.

[13] H. Xie, J. Wang, T. Xi, and Y. Liu, "Thermal conductivity of suspensions containing nanosized $\mathrm{SiC}$ particles," International Journal of Thermophysics, vol. 23, no. 2, pp. 571580, 2002.

[14] S. Murshed, K. Leong, and C. Yang, "Enhanced thermal conductivity of $\mathrm{TiO}_{2}$ - water based nanofluids," International Journal of Thermal Sciences, vol. 44, no. 4, pp. 367-373, 2005.

[15] S. Murshed, K. Leong, and C. Yang, "Investigations of thermal conductivity and viscosity of nanofluids," International Journal of Thermal Sciences, vol. 47, no. 5, pp. 560-568, 2008.

[16] B. Yang and Z. Han, "Temperature-dependent thermal conductivity of nanorod-based nanofluids," Applied Physics Letters, vol. 89, no. 8, p. 083111, 2006.

[17] X. Zhang, H. Gu, and M. Fujii, "Effective thermal conductivity and thermal diffusivity of nanofluids containing spherical and cylindrical nanoparticles," Journal of Applied Physics, vol. 100, no. 4, p. $044325,2006$.

[18] X. Zhang, H. Gu, and M. Fujii, "Effective thermal conductivity and thermal diffusivity of nanofluids containing spherical and cylindrical nanoparticles," Experimental Thermal and Fluid Science, vol. 31, no. 6, pp. 593-599, 2007.

[19] E. Yamada and T. Ota, "Effective thermal conductivity of dispersed materials," Heat and Mass Transfer, vol. 13, no. 1, pp. 27-37, 1980.

[20] J. Koo, Y. Kang, and C. Kleinstreuer, "A nonlinear effective thermal conductivity model for carbon nanotube and nanofiber suspensions," Nanotechnology, vol. 19, no. 37, p. $375705,2008$.
[21] E. V. Timofeeva, J. L. Routbort, and D. Singh, "Particle shape effects on thermophysical properties of alumina nanofluids," Journal of Applied Physics, vol. 106, no. 1, p. 014304, 2009.

[22] M. M. Ghosh, S. Roy, S. K. Pabi, and S. Ghosh, "A molecular dynamics-stochastic model for thermal conductivity of nanofluids and its experimental validation," Journal of Nanoscience and Nanotechnology, vol. 11, no. 3, pp. 2196-2207, 2011.

[23] M. Ghosh, S. Ghosh, and S. Pabi, "Effects of particle shape and fluid temperature on heat-transfer characteristics of nanofluids," Journal of Materials Engineering and Performance, vol. 22, no. 6, pp. 1525-1529, 2013.

[24] H. Jiang, Q. Xu, C. Huang, and L. Shi, “The role of interfacial nanolayer in the enhanced thermal conductivity of carbon nanotube-based nanofluids," Applied Physics A, vol. 118, no. 1, pp. 197-205, 2015.

[25] L. Yang, X. Xu, W. Jiang, and K. Du, "A new thermal conductivity model for nanorod-based nanofluids," Applied Thermal Engineering, vol. 114, pp. 287-299, 2017.

[26] L. Yang, K. Du, and X. Zhang, "A theoretical investigation of thermal conductivity of nanofluids with particles in cylindrical shape by anisotropy analysis," Powder Technology, vol. 314, p. 328, 2017.

[27] L. Yang and X. Xu, "A renovated Hamilton-Crosser model for the effective thermal conductivity of CNTs nanofluids," International Communications in Heat and Mass Transfer, vol. 81, pp. 42-50, 2017.

[28] J.-Z. Lin, Y. Xia, and X.-K. Ku, "Flow and heat transfer characteristics of nanofluids containing rod-like particles in a turbulent pipe flow," International Journal of Heat and Mass Transfer, vol. 93, pp. 57-66, 2016.

[29] F. Yuan, J. Lin, and X. Ku, "Convective heat transfer and resistance characteristics of nanofluids with cylindrical particles," Heat Transfer Engineering, vol. 39, no. 6, pp. 1-10, 2018.

[30] J. Buongiorno, "Convective transport in nanofluids," Journal of Heat Transfer, vol. 128, no. 3, pp. 240-250, 2006.

[31] G. Batchelor, "Slender-body theory for particles of arbitrary cross-section in stokes flow," Journal of Fluid Mechanics, vol. 44, no. 3, pp. 419-440, 1970.

[32] M. B. Mackaplow and E. S. Shaqfeh, "A numerical study of the rheological properties of suspensions of rigid, non-Brownian fibres," Journal of Fluid Mechanics, vol. 329, no. 1, pp. 155-186, 1996.

[33] H. Brinkman, "The viscosity of concentrated suspensions and solutions," Journal of Chemical Physics, vol. 20, no. 4, p. 571, 1952.

[34] G. Batchelor, "The effect of Brownian motion on the bulk stress in a suspension of spherical particles," Journal of Fluid Mechanics, vol. 83, no. 1, pp. 97-117, 1977.

[35] S. Ferrouillat, A. Bontemps, O. Poncelet, O. Soriano, and J.-A. Gruss, "Influence of nanoparticle shape factor on convective heat transfer and energetic performance of waterbased $\mathrm{SiO}_{2}$ and $\mathrm{ZnO}$ nanofluids," Applied thermal engineering, vol. 51, no. 1, pp. 839-851, 2013.

[36] S. G. Advani and C. L. Tucker, "The use of tensors to describe and predict fiber orientation in short fiber composites," Journal of Rheology, vol. 31, no. 8, pp. 751-784, 1987.

[37] J.-Z. Lin and Z.-J. You, "Stability analysis in spatial mode for channel flow of fiber suspensions," Applied Mathematics and Mechanics-English Edition-Shanghai, vol. 24, no. 8, pp. 871879, 2003.

[38] Z. J. You and J. Z. Lin, "Stability in the circular pipe flow of fiber suspensions," Journal of Hydrodynamics, vol. 15, no. 2, pp. 12-18, 2003. 
[39] J. S. Cintra and C. L. Tucker, "Orthotropic closure approximations for flow-induced fiber orientation," Journal of Rheology, vol. 39, no. 6, pp. 1095-1122, 1995.

[40] F. Folgar and C. L. Tucker, "Orientation behavior of fibers in concentrated suspensions," Journal of Reinforced Plastics and Composites, vol. 3, no. 2, pp. 98-119, 1984.

[41] J. G. de la Torre and V. A. Bloomfield, "Hydrodynamic properties of complex, rigid, biological macromolecules: theory and applications," Quarterly Reviews of Biophysics, vol. 14 , no. 1, p. 81, 1981.

[42] G. Li and J. X. Tang, "Diffusion of actin filaments within a thin layer between two walls," Physical Review E, vol. 69, no. 6, p. 061921, 2004.

[43] J. A. Olson, "The motion of fibres in turbulent flow, stochastic simulation of isotropic homogeneous turbulence," International Journal of Multiphase Flow, vol. 27, no. 12, pp. 2083-2103, 2001.

[44] Z. You and J. Lin, "Effects of tensor closure models and 3-D orientation on the stability of fiber suspensions in a channel flow," Applied Mathematics and Mechanics, vol. 26, no. 3, pp. 307-312, 2005.

[45] S. K. Friedlander, Smoke, Dust, and Haze: Fundamentals of Aerosol Dynamics, Oxford University Press, New York, NY, USA, 2000. 


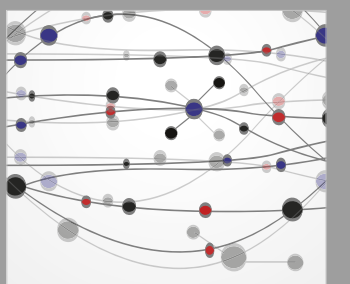

The Scientific World Journal
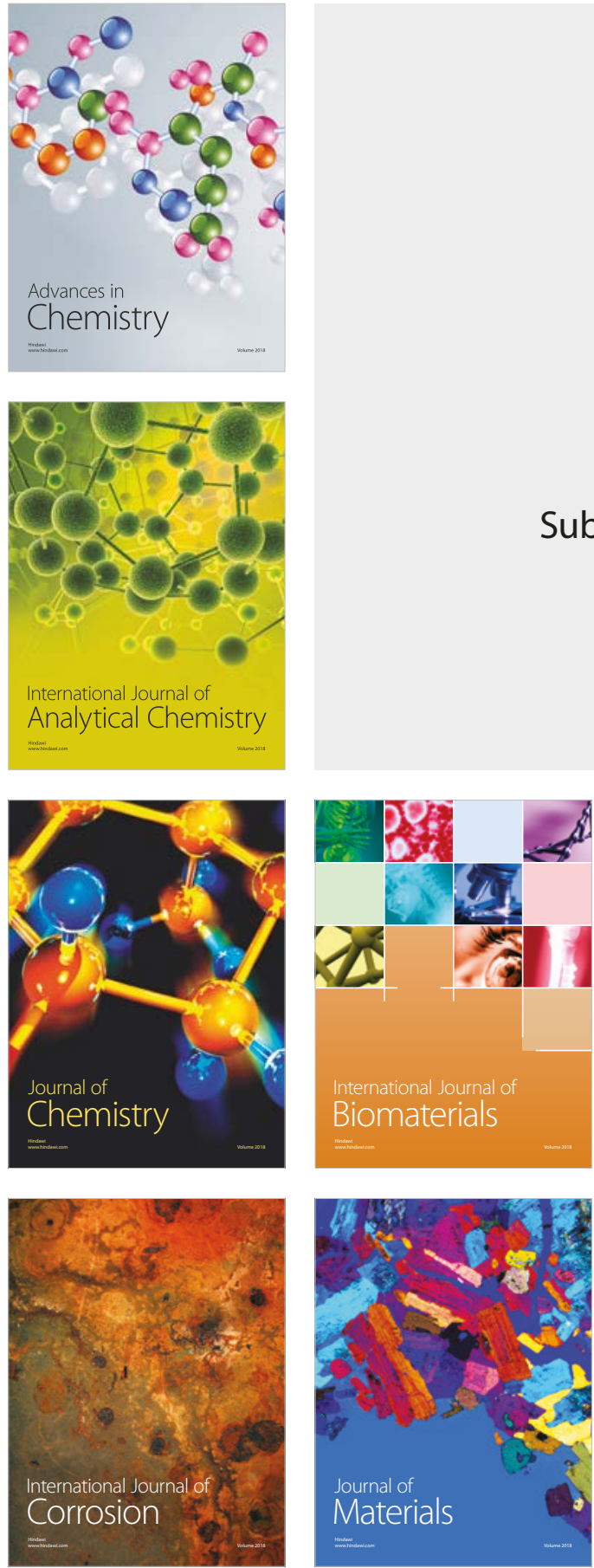

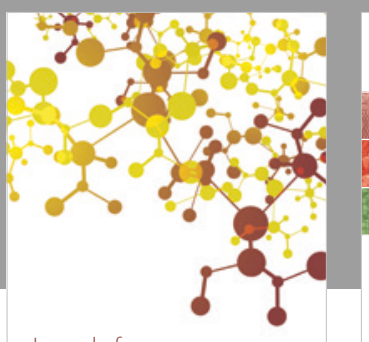

Journal of

Applied Chemistry
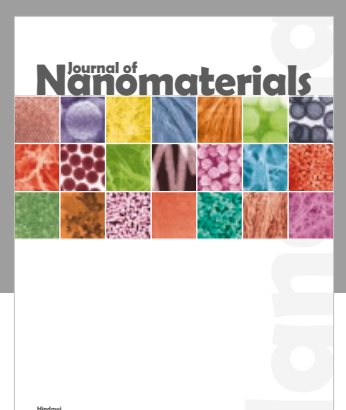

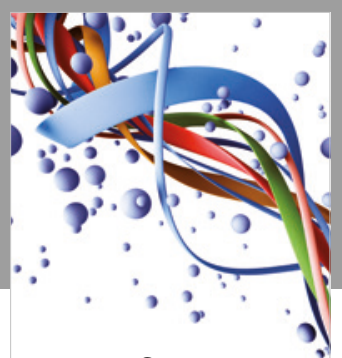

Scientifica

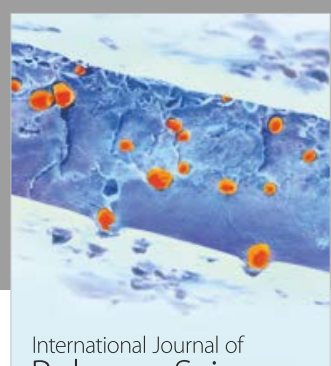

Polymer Science

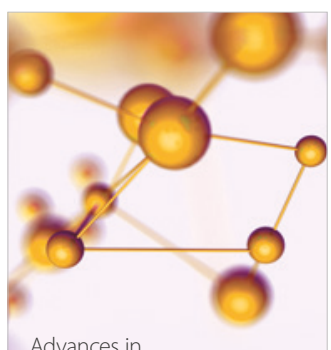

Physical Chemistry
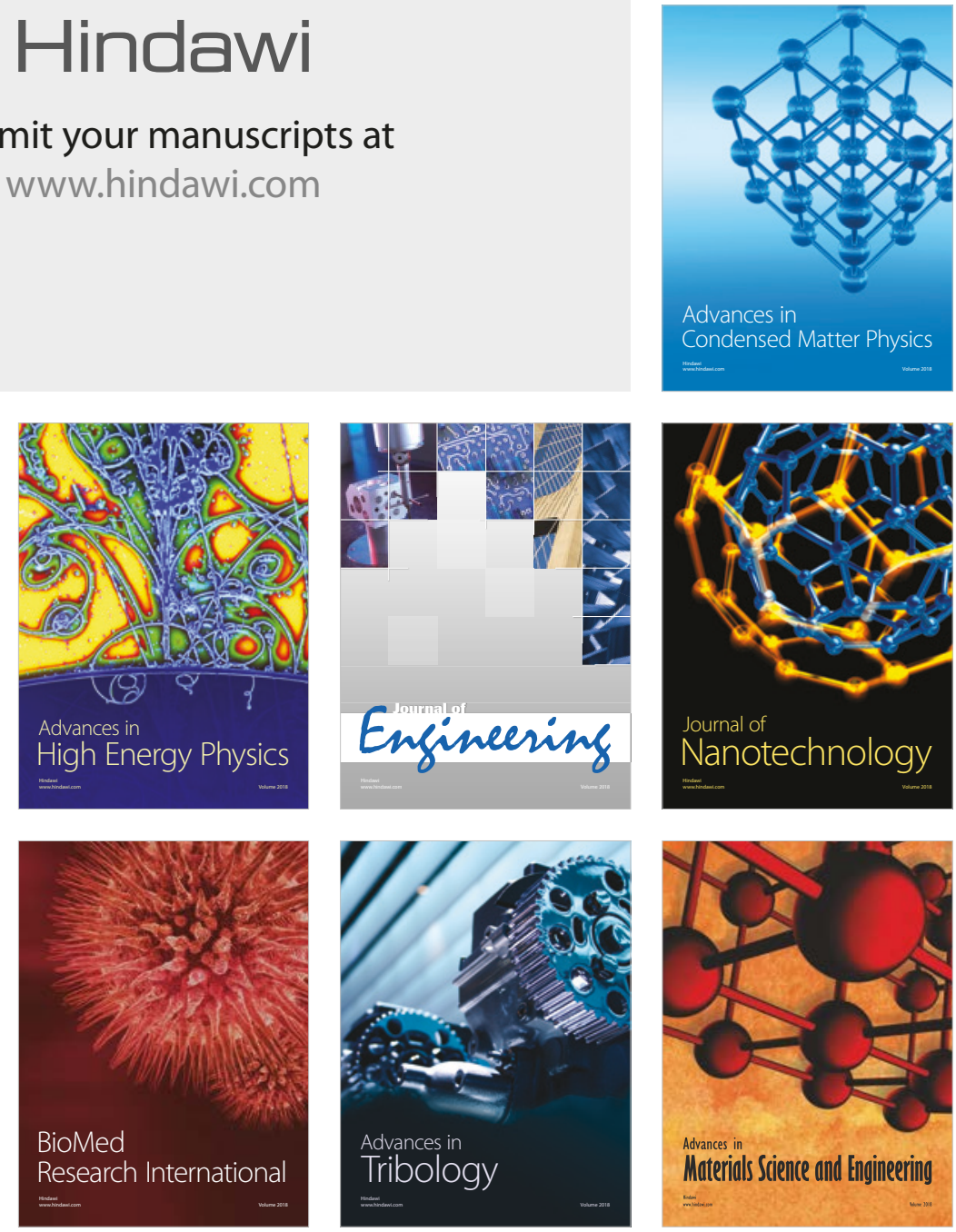